\title{
Erratum to: The new WHO classification of ovarian, fallopian tube, and primary peritoneal cancer and its clinical implications
}

\author{
Ivo Meinhold-Heerlein ${ }^{1}$ Christina Fotopoulou ${ }^{2,3}$ - Philipp Harter ${ }^{4}$. \\ Christian Kurzeder $^{4}$ - Alexander Mustea ${ }^{5}$ Pauline Wimberger ${ }^{6}$. \\ Steffen Hauptmann ${ }^{7} \cdot$ Jalid Sehouli $^{2}$
}

Published online: 13 April 2016

(C) Springer-Verlag Berlin Heidelberg 2016

\section{Erratum to: Arch Gynecol Obstet 293:695-700 DOI 10.1007/s00404-016-4035-8}

Unfortunately, the affiliations of the coauthors are not displayed correctly in the online publication.

Details of affiliation are:

Christina Fotopoulou: University Hospital Charité Berlin, Berlin, Germany and Imperial College NHS Trust, London, England, UK

Philipp Harter: Kliniken Essen-Mitte, Essen, Germany

Christian Kurzeder: Kliniken Essen-Mitte, Essen, Germany Alexander Mustea: University Hospital Greifswald, Greifswald, Germany

Pauline Wimberger: University Hospital Dresden, Dresden, Germany

The online version of the original article can be found under doi:10.1007/s00404-016-4035-8.

Ivo Meinhold-Heerlein

imeinhold@ukaachen.de

1 Klinik für Gynäkologie und Geburtsmedizin, Uniklinik RWTH Aachen, Pauwelsstr. 30, 52074 Aachen, Germany

2 University Hospital Charité Berlin, Berlin, Germany

3 Imperial College NHS Trust, London, England, UK

4 Kliniken Essen-Mitte, Essen, Germany

5 University Hospital Greifswald, Greifswald, Germany

6 University Hospital Dresden, Dresden, Germany

7 University Hospital Maastricht, Maastricht, The Netherlands
Steffen Hauptmann: University Hospital Maastricht, The Netherlands

Jalid Sehouli: University Hospital Charité Berlin, Berlin, Germany 